\title{
CONFERENCE PHOTOS
}


This page is intentionally left blank 


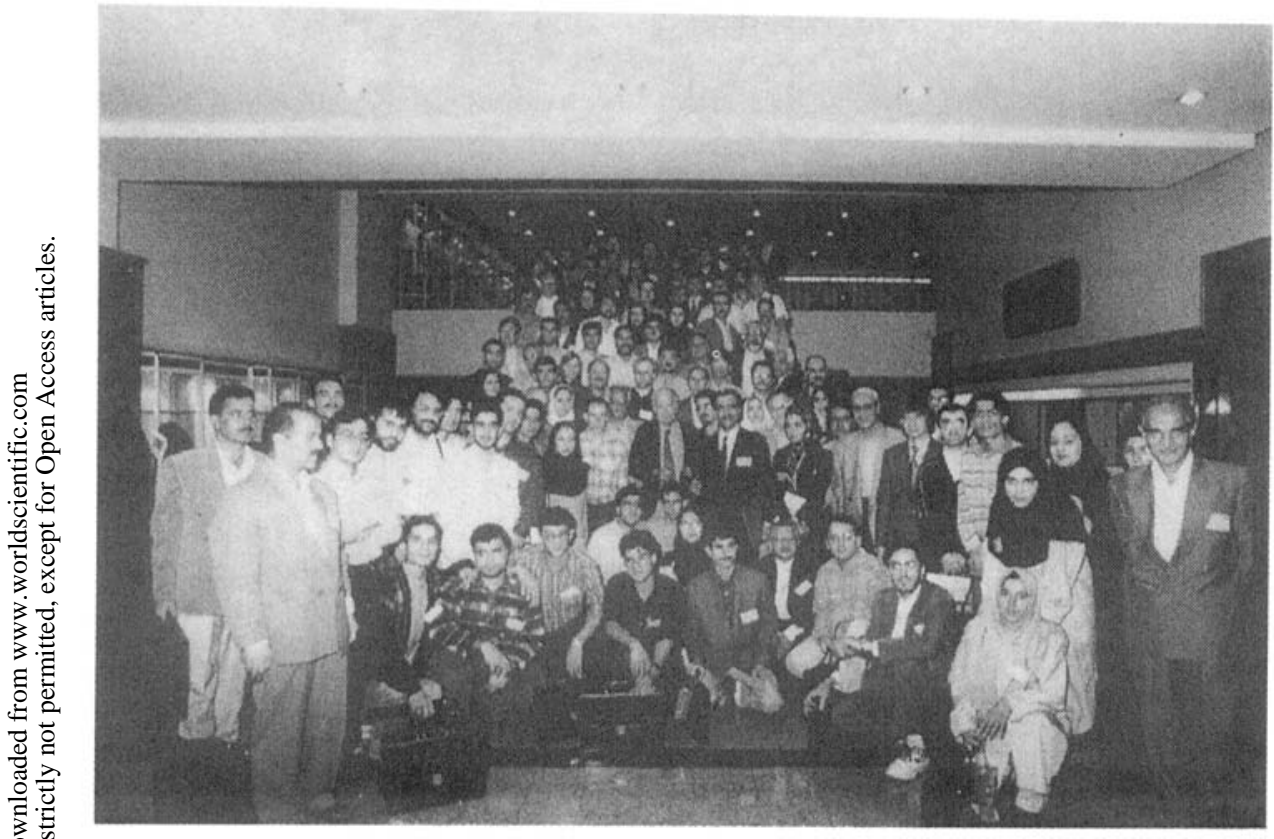

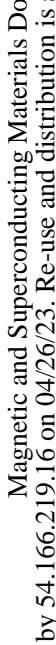

Participants at the First Regional Conference on Magnetic and Superconducting Materials(MSM-99)

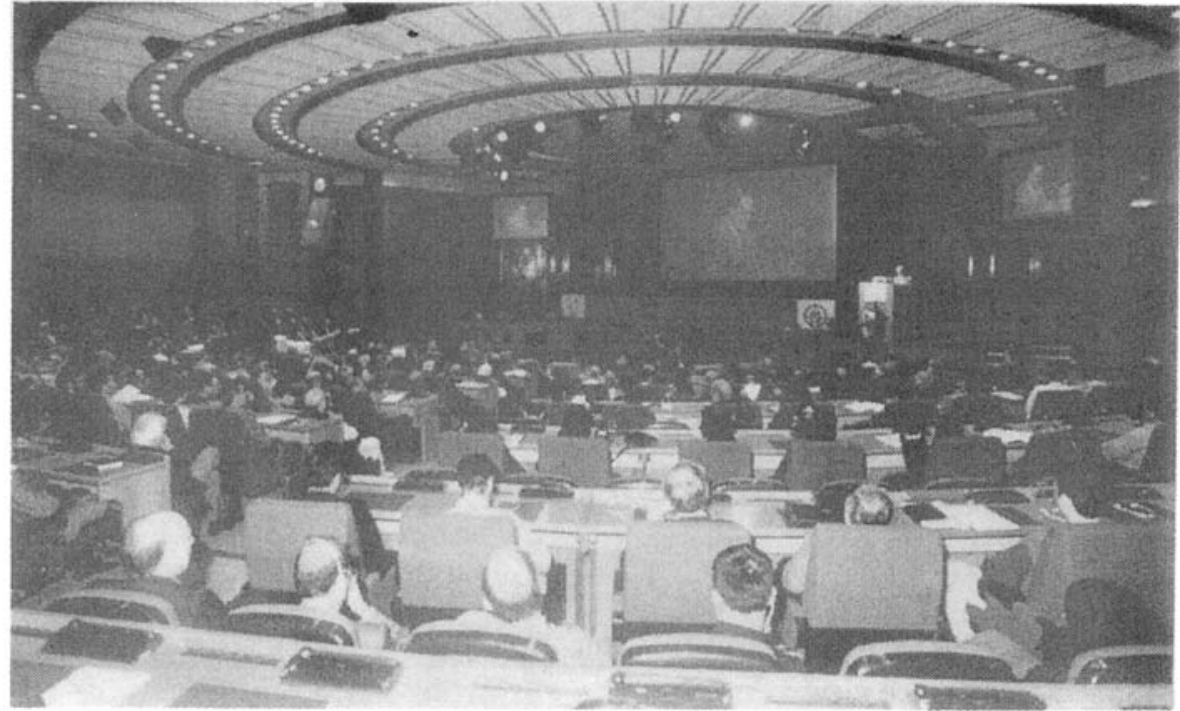

MSM-99 Conference view 


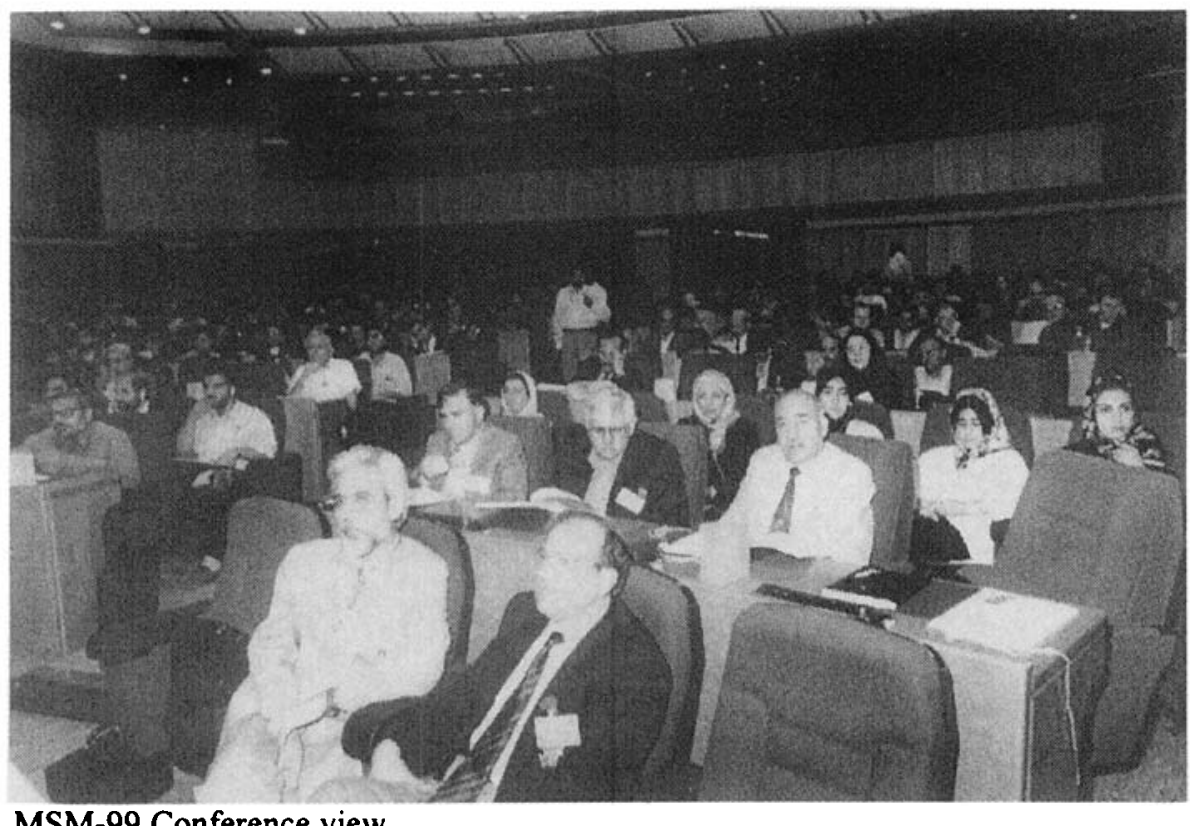

\section{MSM-99 Conference view}

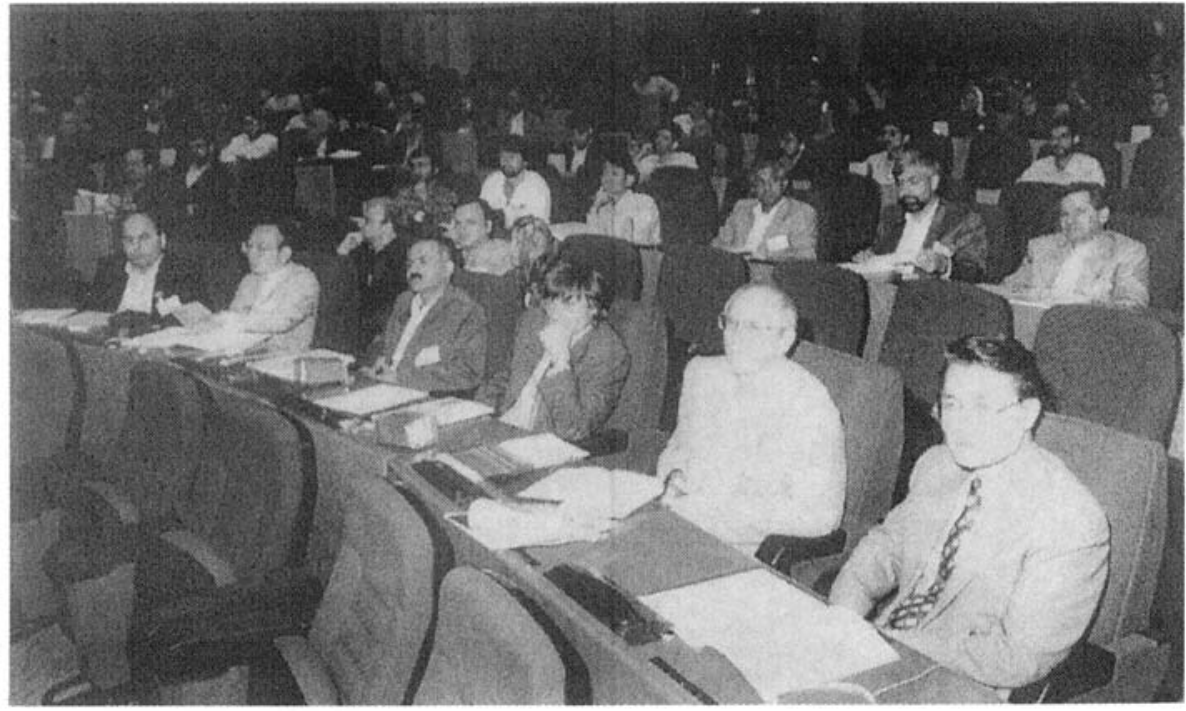

MSM-99 Conference view 


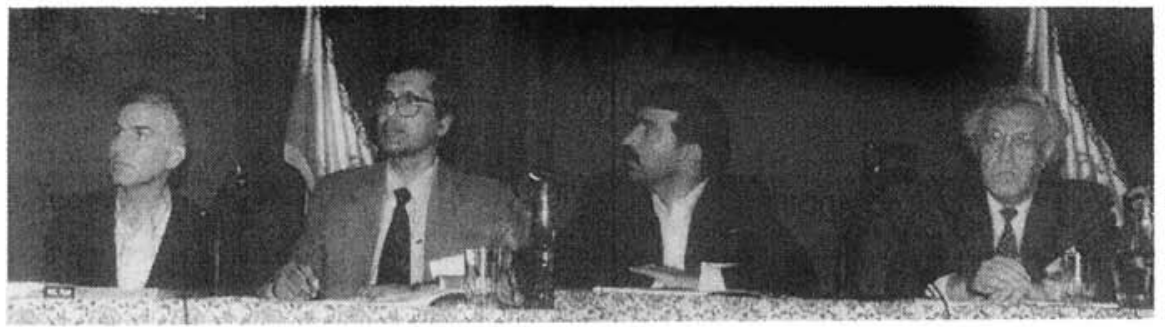

A.Z. Moshfegh, K.M.N.A. Islam (Chairs) H. Arabi and V. Aroutiounain (Chairs)

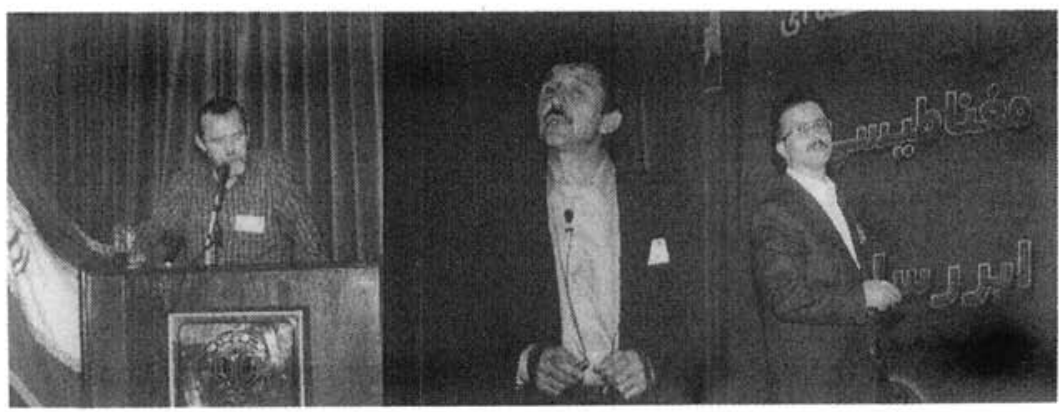

J. Jensen

A.V. Bondarenko V. Daadmehr

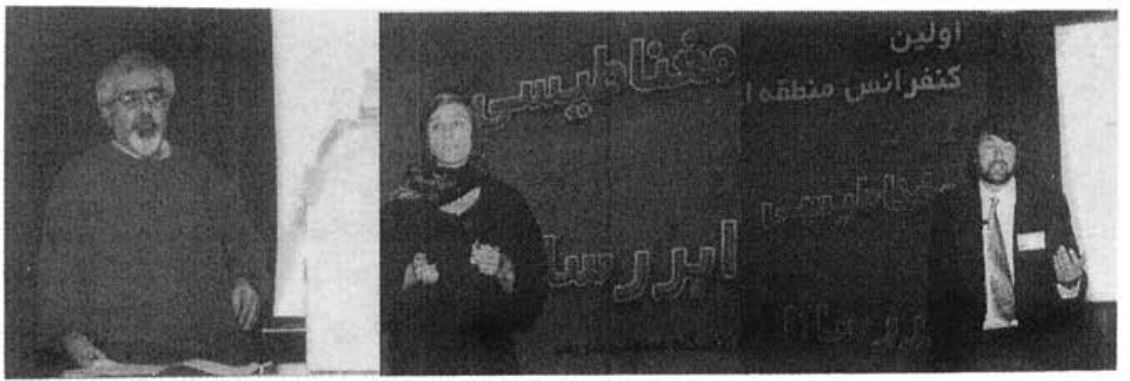

M.A. Vesaghi

C. Ambroch-Draxl

P. Hatton

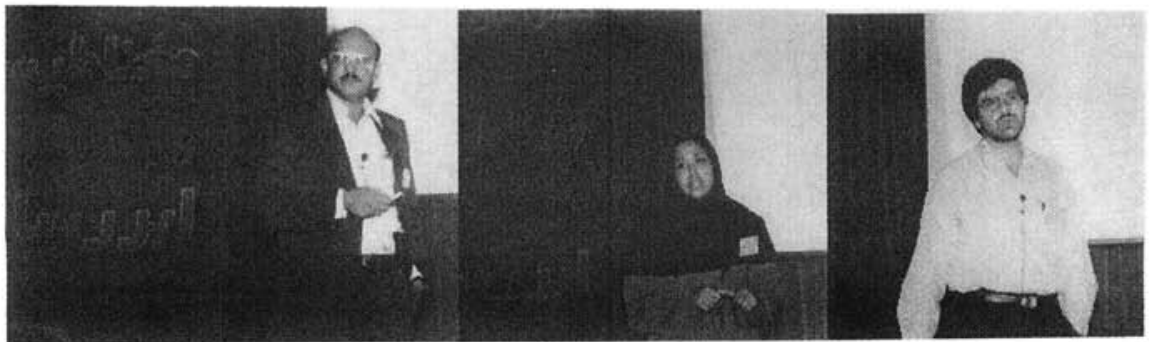

S.K. Hassanain

B. Chanda

M.R. Mohammadi zadeh 


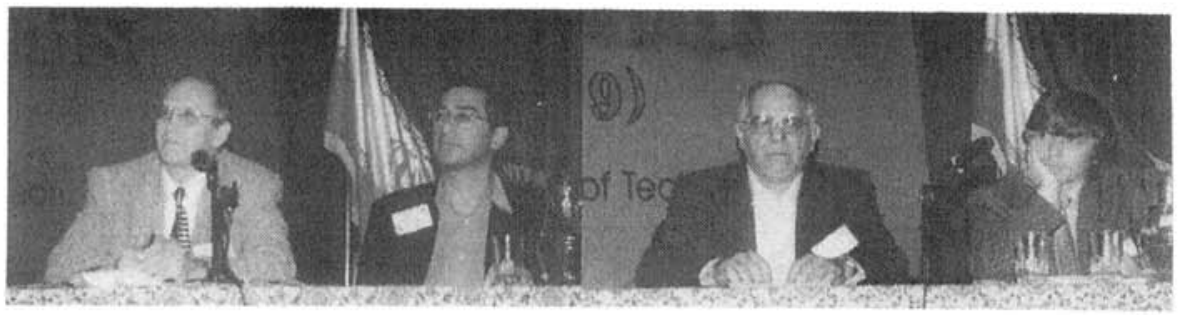

Yu.D. Tretyakov and K. Behnia (Chairs)

M. Zargar-Shooshtari and E. Antipov (Chairs)

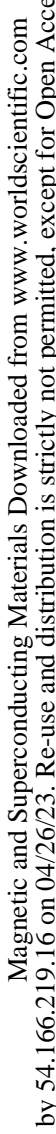

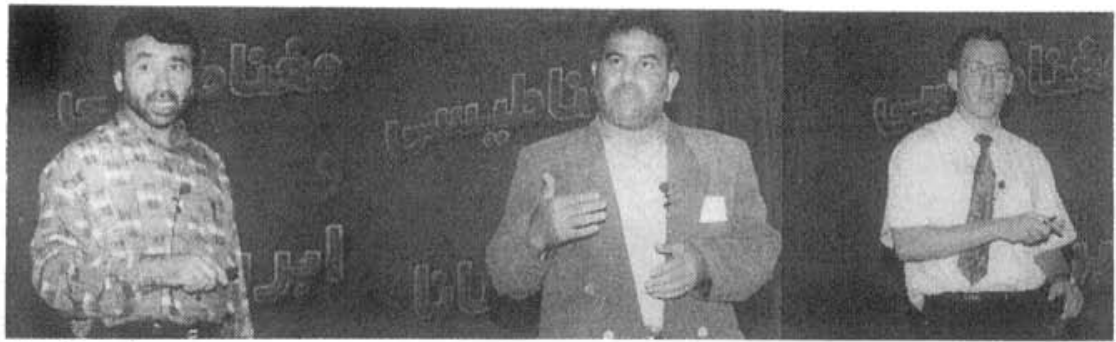

M. Ghazi

M. Farbod

S. Dorbolo

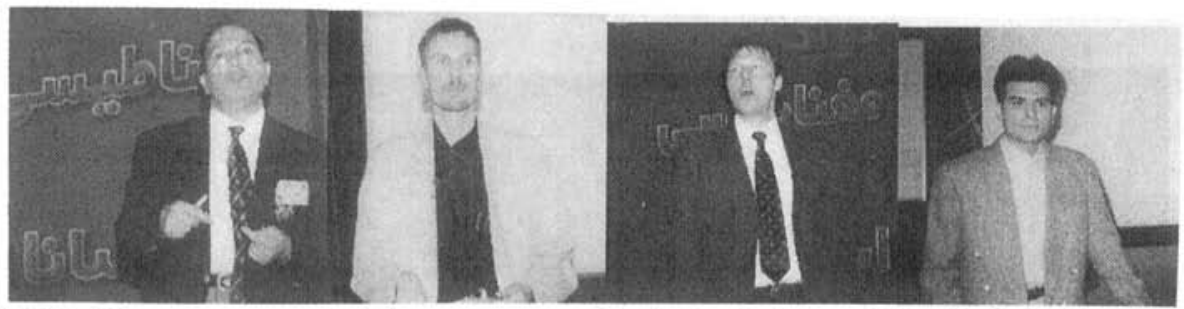

M.N. Khan

J. Hissa

C. Nadarzinski

H.R. Madaah-Hosseini

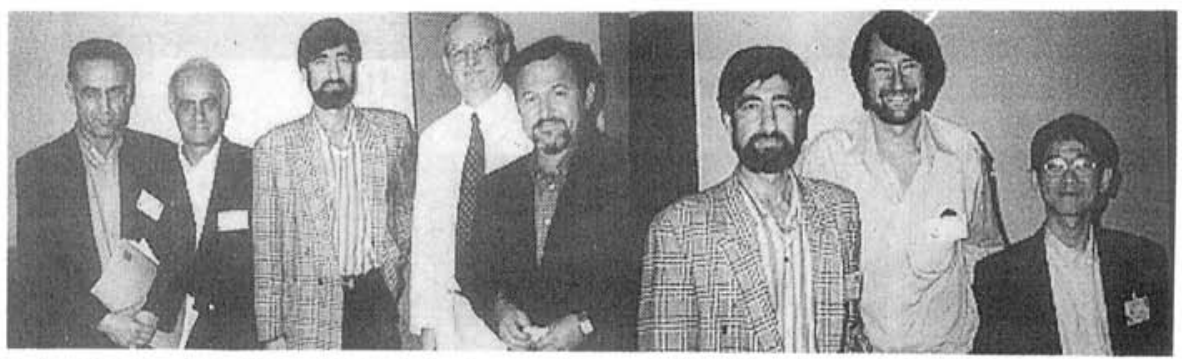

J. Amighian, M. Safa, A.M. Ghorbanzadeh, A.M. Ghorbanzadeh, P.D. Hotton

P. Goernert and A. Kiyanvash at tea break. and C.H. Du at tea break. 


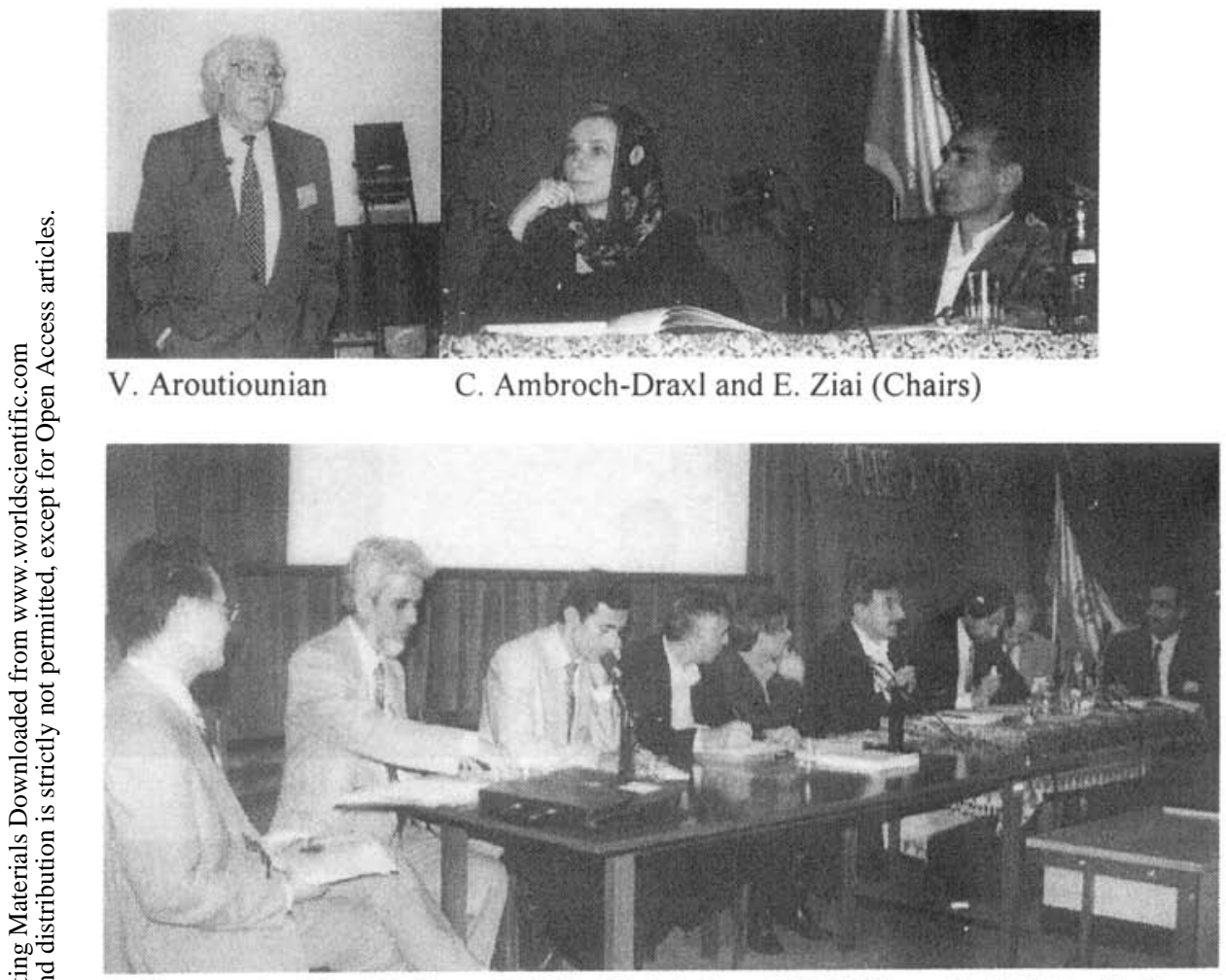

K. Kitazawa, A. ul-Haq, S. Mahmood, A. Moshfegh, A. Yousefi, H.U. Habermeier, P. Hatton, Yu.D. Tretyakov and M. Akhavan at panel discussion session on scientific network

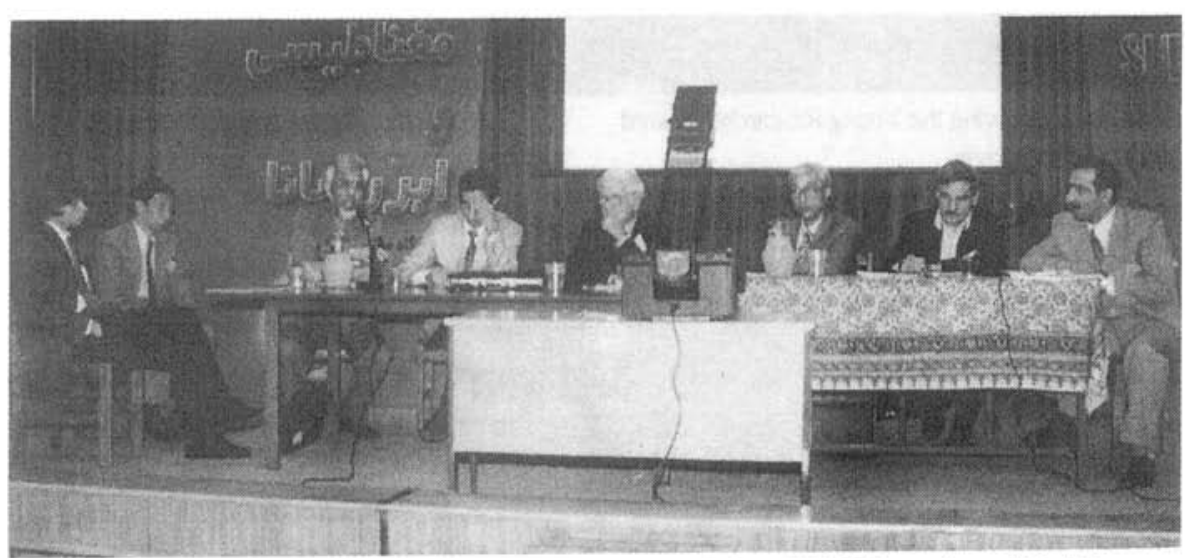

E. Antipov, L. Yu, A. ul-Haq, S. Mahmood, A. Barone, A. Mookerjee, H.U. Habermeier and M. Akhavan at panel discussion session on science and technology 


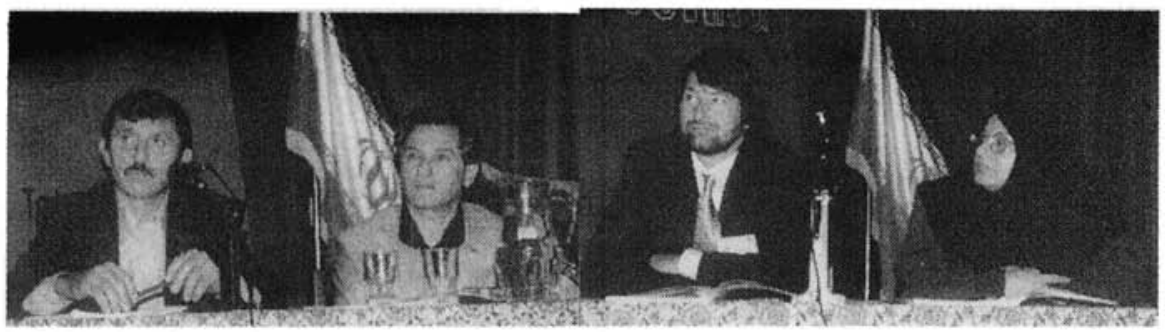

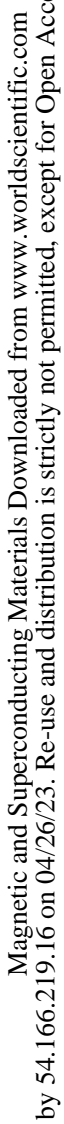

A.V. Bondarenko and A. Yousefi (Chairs)

P. Hatton and A. Iraji zad (Chairs)

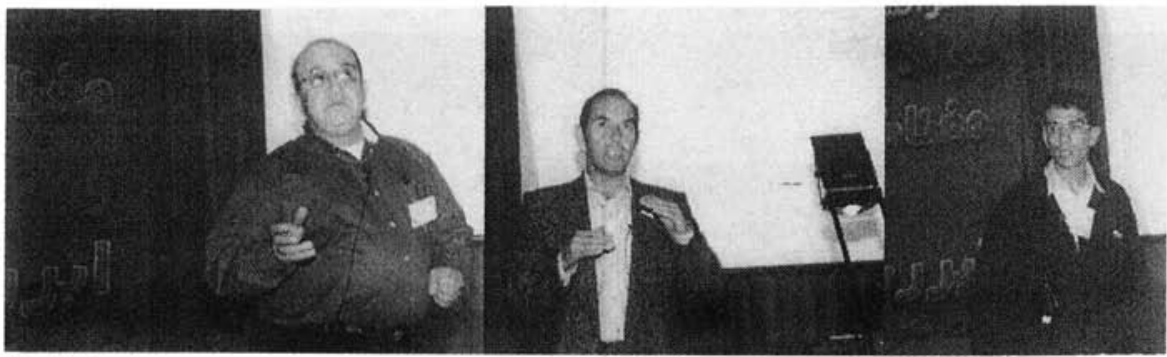

D. Fathy

Yu.M. Shukrinov

M. Kohandel

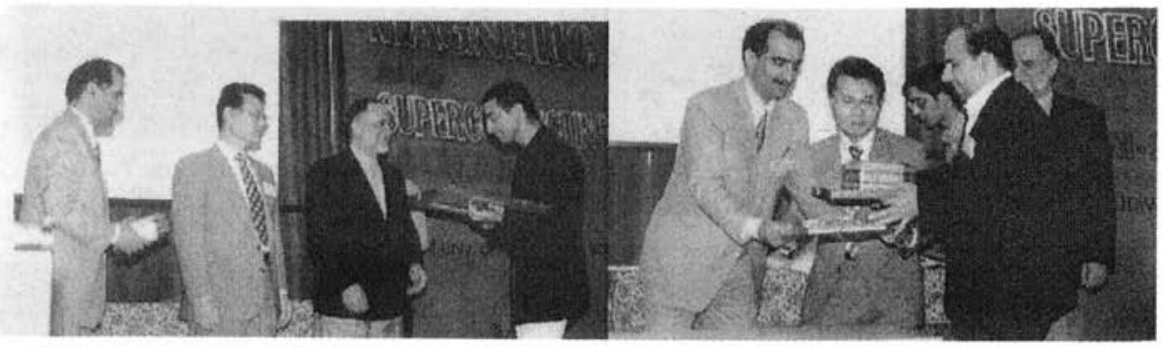

K. Behnia, receiving the Young Researcher Award J.B. Jamali, receiving the Young Researcher in superconductivity Award in magnetism

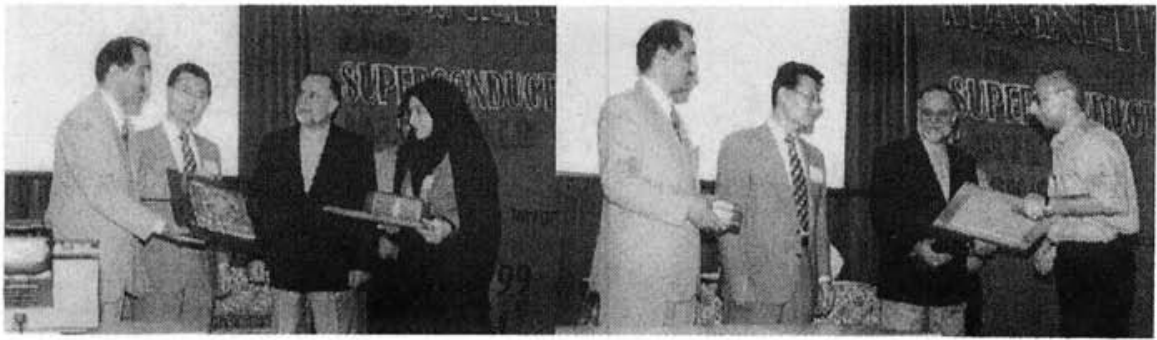

H. Shakeripour, receiving the Best Poster Award

A. Nader, receiving the Best Poster Award in superconductivity in superconductivity 


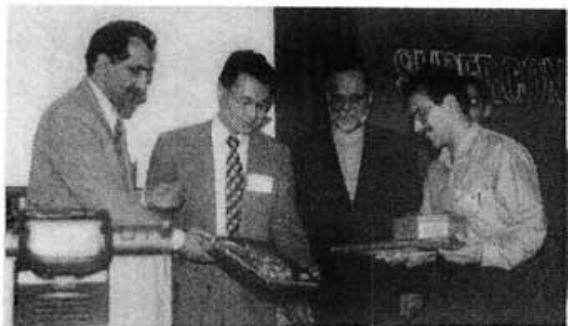

M. Ghominezhad, receiving the Best Poster Award in magnetism

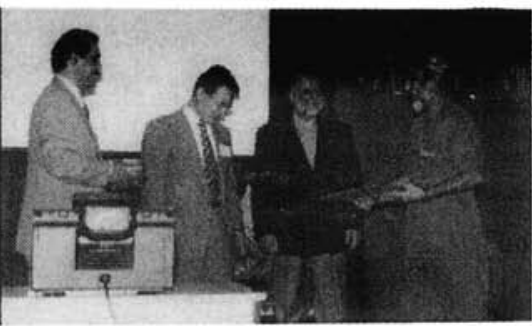

A. ul-Haq, receiving the Best Poster Award in magnetism for Z. Ahmad

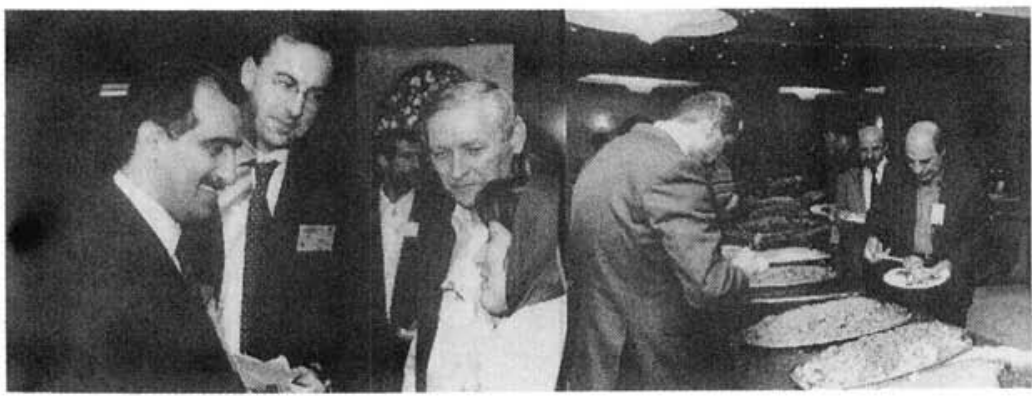

M. Akhavan, F. Tellier, D. Fruchart and Participants at lunch R.M. Galera at reception

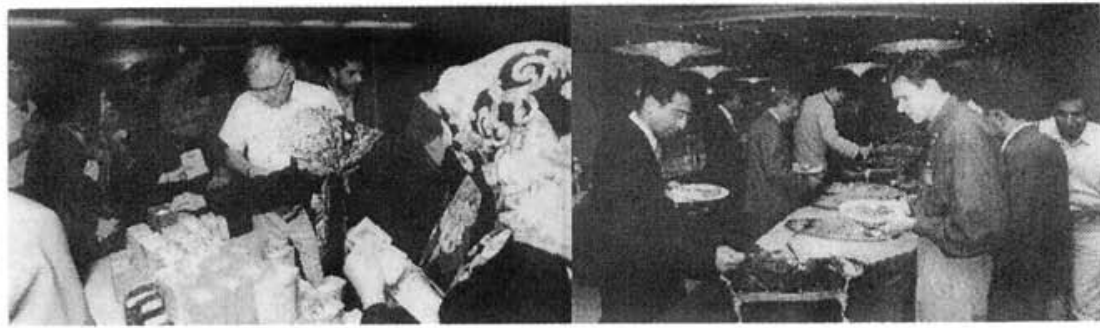

Participants taking conf. souvenir (mug) Participants at lunch

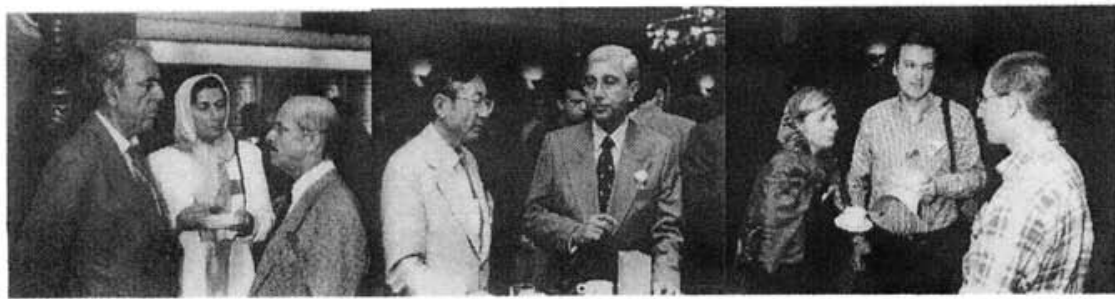

H. Yaghoubi, M.

Dehghani, T. Eliasi-Rad at reception
N. Achiwa and A.

Mookerjee

at reception
C. Ambrosch-Draxl, K.E. Ambrosch and S. Dorbolo at reception 


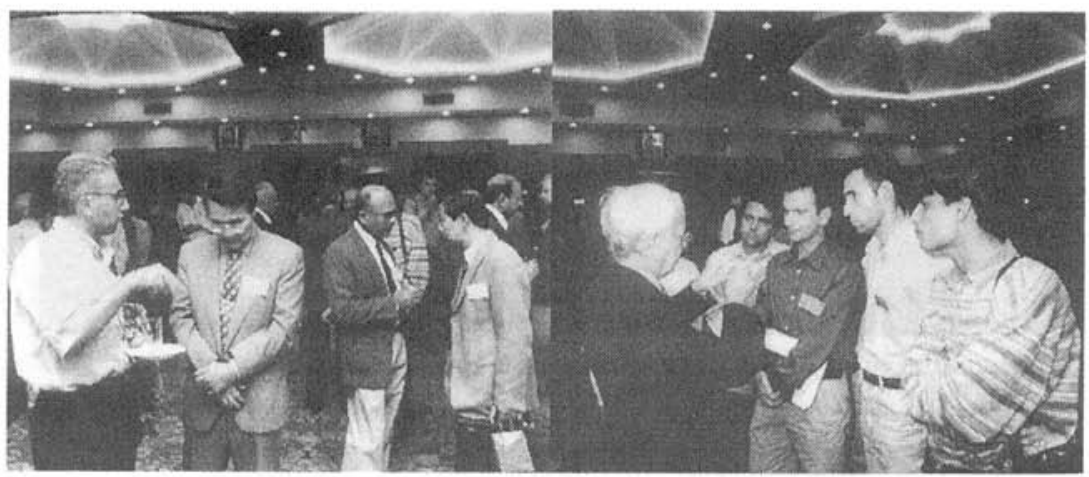

A. Nader, K. Kitazawa, G. Baskaran and L. Yu A. Barone with students at reception

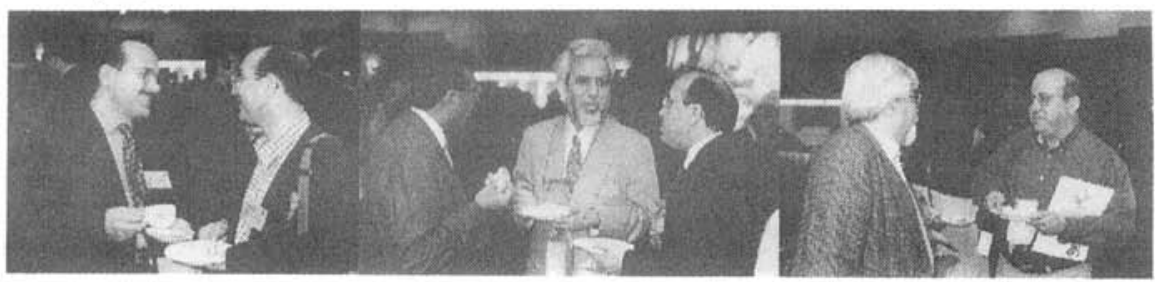

A. Ostad-Hosseini and A. I. Ahmad, A. ul-Haq and Shafiekhani at reception M.N. Khan at reception M. Vesaghi and D. Fathy

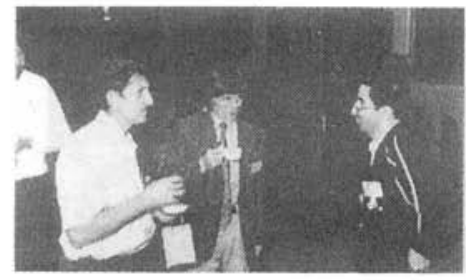

A.V. Bondarenko, E. Antipiov, A.M. Aliev at reception

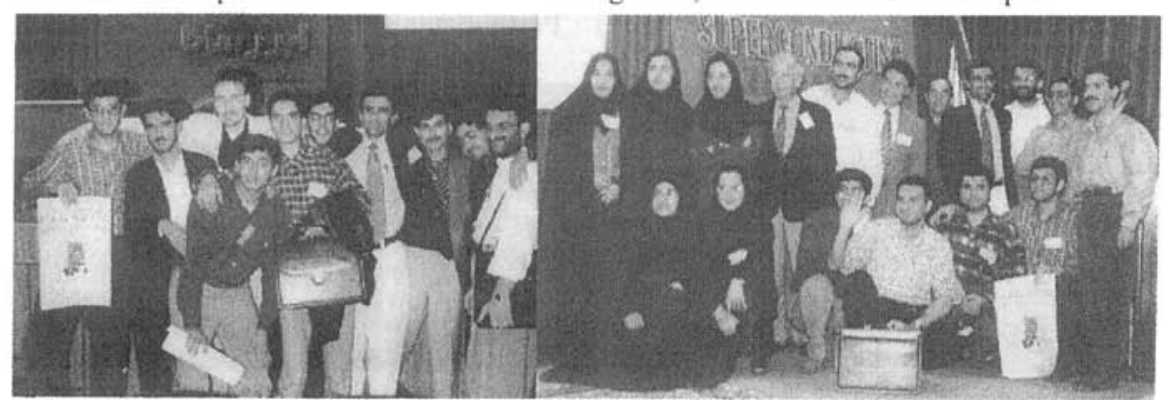

J. Hissa and S. Mahmood with students

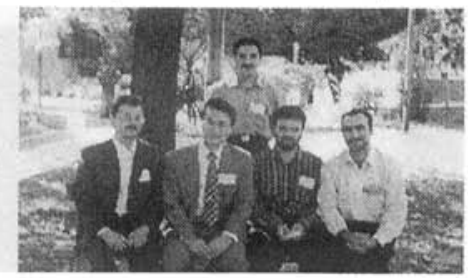

V. Daadmehr, K. Kitazawa, M. Ghominezhad, H. Naghshara, P. Sahebsara on Univ. Campus

A. Barone, K. Kitazawa, Y.M. Shuikrinov, S. Mahmood with students 


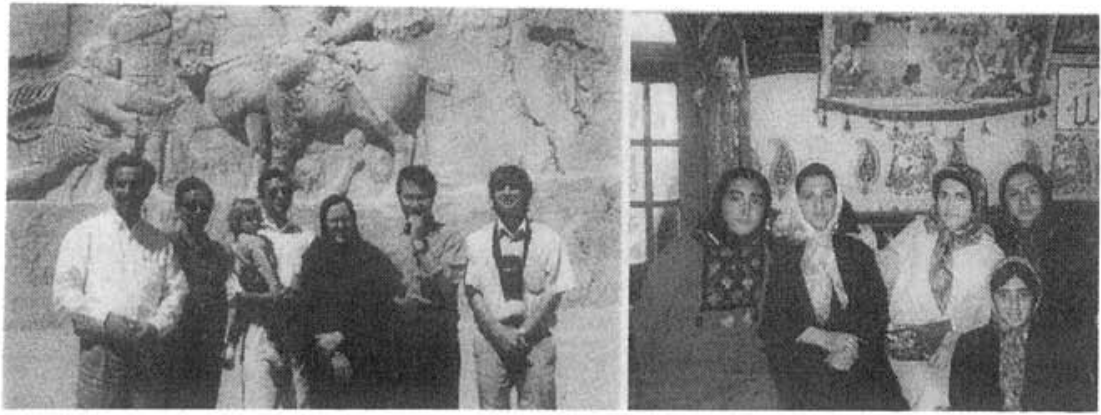

M. Akhavan, L. Yu, T. Mahmood, S. Mahmood, L.

Conf. staff at a tea house, Isfahan

MoCann, K. Kitazawa, E. Antipov at Takhte-Rostam, Shiraz

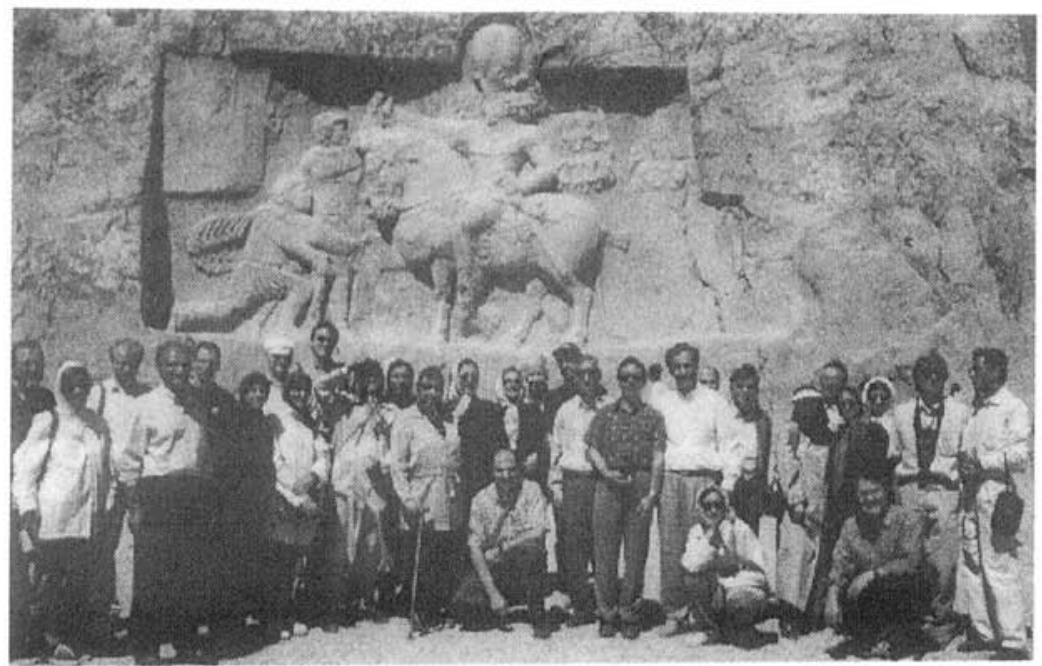

Participants at Takhte-Rostam, Shiraz

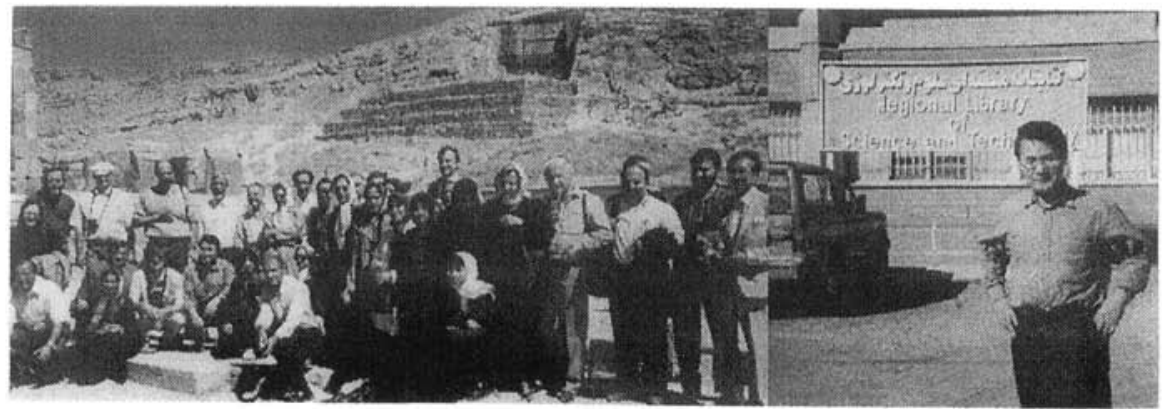

Participants in the post-conf. tour at Persepolis, Shiraz

K. Kitazawa at the Regional Lib. of Sci. \& Tech., Shiraz University 


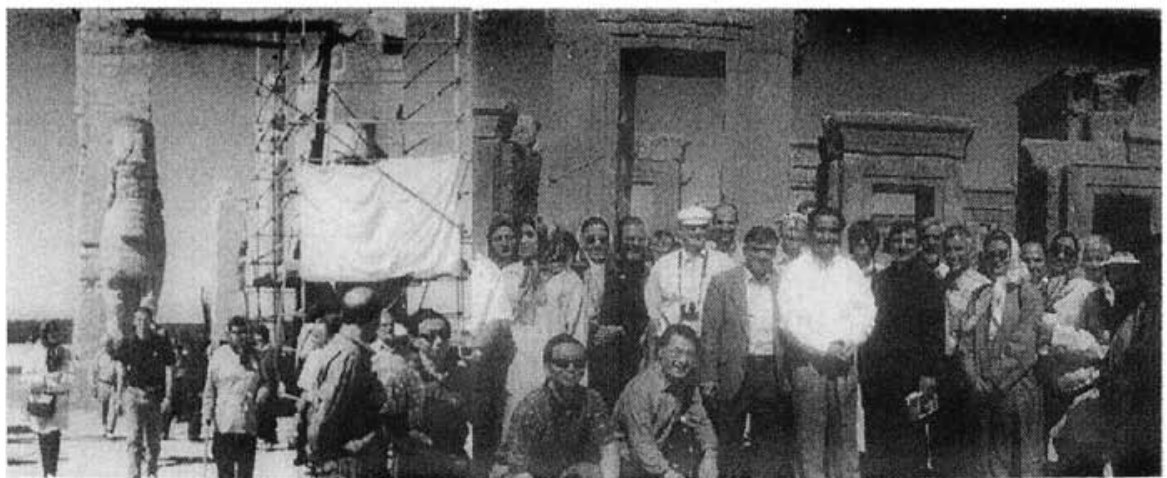

Participants in the post-conf. tour at Persepolis, Shiraz

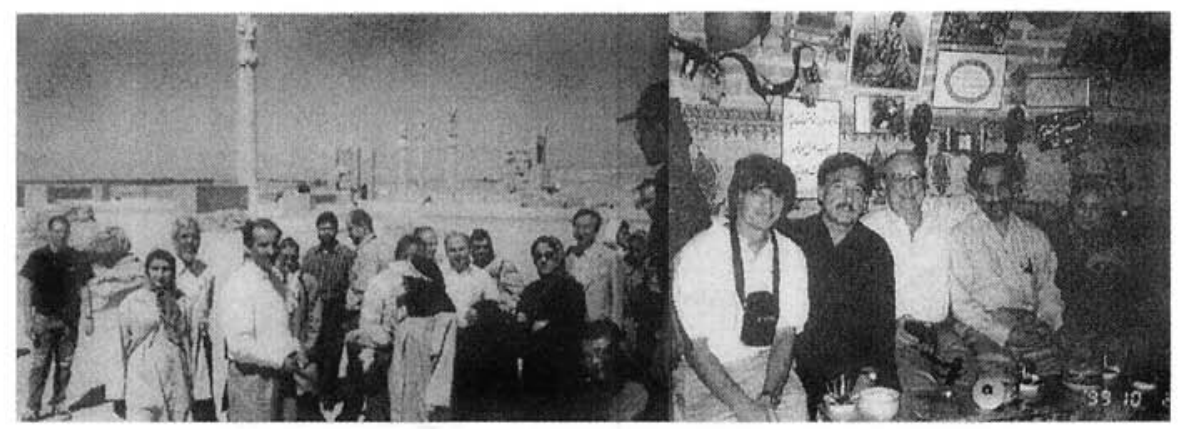

Participants in the post-conf. tour at Persepolis, Shiraz

E. Antipov, H.U. Habermeire, P. Goemert, M. Akhavan, F. Tabassian at a tea house, Isfahan

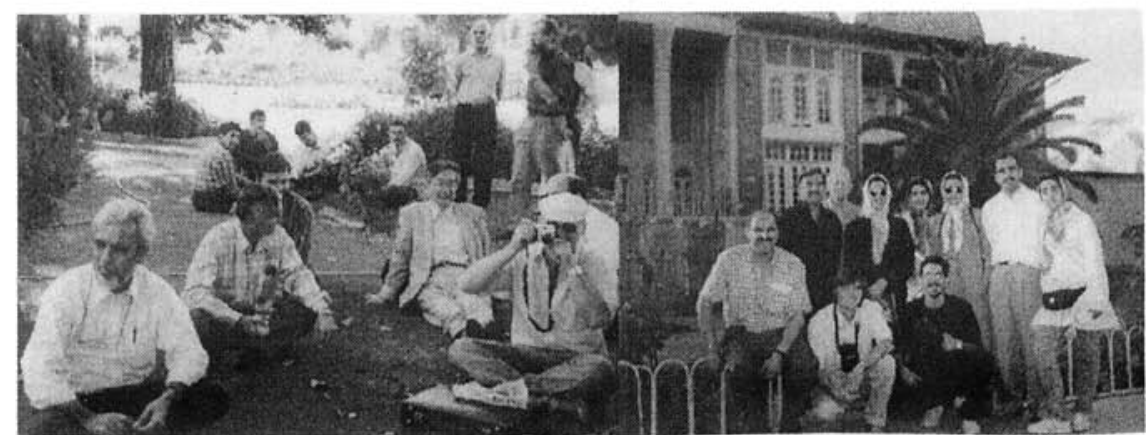

Participants relaxing at Eram Garden, Shiraz

Participants at Eram Garden, Shiraz 


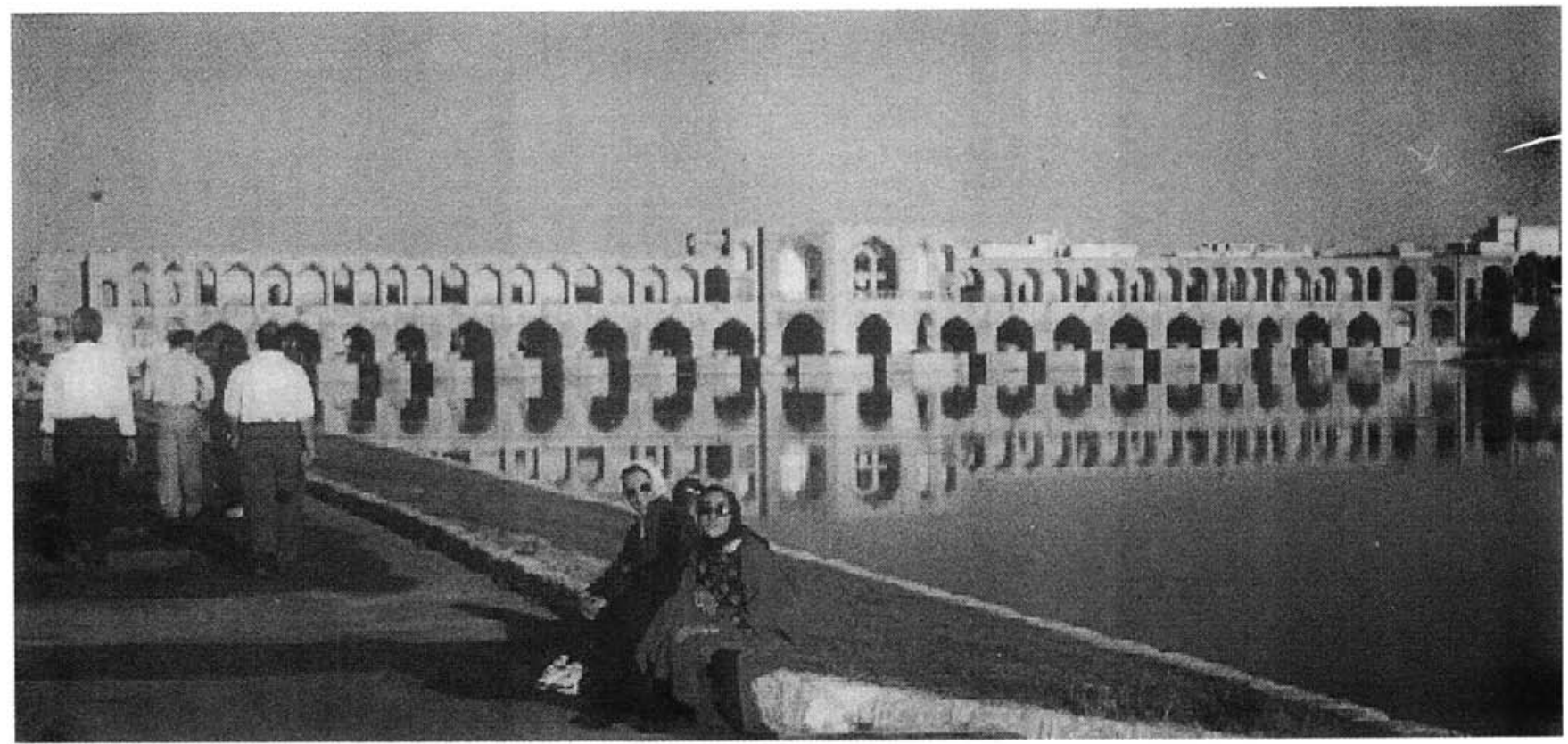

Participants in the post-conf. tour at Sio-Seh Pol, Isfahan 


\section{AUTHOR INDEX}

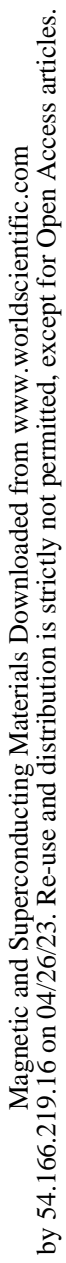


This page is intentionally left blank 


\begin{tabular}{|c|c|c|c|c|c|}
\hline Aassit, E.L. & 536,593 & Arouri, T.R. & 447 & Cheranorskï, v.O. & 893 \\
\hline Abakumov, A.M. & 137 & Aroutiounian, V.M. & 653,661 & Chemy, A.S. & 801 \\
\hline Abbas, T. & 1068 & Askerzade, I.N. & 107 & Cherpak, N.T. & 677 \\
\hline Abrahamsen, A.B. & 476 & Ataev, B.M. & $195,581,961$ & Chioncel, L. & 727 \\
\hline Abdukragidov, Sh.8 & 961 & Austoos, $M$. & 341 & Chu, W.K. & 601 \\
\hline Abu-Ajarayesh, I. & 783,1115 & Babaei, A.R. & 842 & Churilov, G.E. & 393 \\
\hline Abu-Talib, I. & 598 & Babaei & & Cobas, $R$. & 541,549 \\
\hline Achiwa, $N$. & 809,817 & Brojeny, A. & 181 & Collins, S.P. & 936 \\
\hline Adroja, D.T. & 943 & Bacmann, M. & 991 & Cristiano, $R$. & 645 \\
\hline Agatonov, O.B. & 393 & Balinskè, L. & 860 & Daadmehr, V. & 175,335 \\
\hline Ahmad, I. & 1027 & Barakat, N.M. & 669 & Dai, J.H. & 51 \\
\hline Alhmad, $Z$. & 1068 & Barone, A & 619,645 & Dartyge, E. & 727 \\
\hline Ahmod, M.M. & 153 & Baskaran, G. & 53,89 & Davies, H.A. & 1027 \\
\hline Ajiro, Y. & 809 & Batdalov, AB. & 961 & Do Biasi, R.S. & 860 \\
\hline Albarzadeh, H. & 251 & Batista- & & Dedek, U. & 499 \\
\hline Akhavan, $M$. & $\begin{array}{l}3,11,161,175 \\
251,335,523 \\
585,1007 \\
1099,1107\end{array}$ & $\begin{array}{l}\text { Leyva, A.J. } \\
\text { Behnia, K. } \\
\text { Beznosov, A.B. } \\
\text { Bishop, D.J. }\end{array}$ & $\begin{array}{l}541,549 \\
229,307 \\
881,1023 \\
433,476\end{array}$ & $\begin{array}{l}\text { Derev'anko, S.A. } \\
\text { Derevyanko, V.V. } \\
\text { Dey, T.K. } \\
\text { Dikin, D.A. }\end{array}$ & $\begin{array}{l}681 \\
312 \\
295 \\
393\end{array}$ \\
\hline Akhavan, 0 . & 585 & Bondarenko, AV. & 447,499 & Dmitriev, V.M. & $393,401,956$ \\
\hline Ali, A & 1068 & Bordet, P. & 137 & & 967 \\
\hline Aliav, A.M. & 961 & Borisenko, V.N. & 499 & Darbolo, S. & 341 \\
\hline Aliov, Kh. K. & 899 & Borowiec, M. & 849 & Dou, S.X. & 319 \\
\hline Alivv, S.A. & 303 & Bougerot & & $\mathrm{Du}, \mathrm{C} \cdot \mathrm{H}$. & 275,936 \\
\hline Aliov, V.M. & 303 & Chaillout, C. & 137 & Dunaevshy, S.M. & 1045 \\
\hline Alouani, M. & 727 & Bougrine, $\mathrm{H}$. & 341 & Dworschak, F. & 499 \\
\hline At-Sharif, A. & 783 & Braysy, V. & 83,145 & Dyakonov, V. & 697,855 \\
\hline Altshuler, E. & 541,549 & Brouder, Ch. & 727 & Dzthurayev, D.R. & $267,3 \pi 7,424$ \\
\hline AL-Zaubi, G.M. & 825 & Brown, S. & 275,936 & & 794,1059 \\
\hline $\begin{array}{l}\text { Ambrosch. } \\
\text { Dray } C .\end{array}$ & 259 & Buckley, R.A & 1027 & Eremenko, V.V. & $\begin{array}{l}237,1023 \\
956\end{array}$ \\
\hline Amighian, J. & 1091 & Buniatyan, V.V. & 653,661 & Eropkin, V.N. & 956,967 \\
\hline Ammendota, G. & 645 & Canfield, P.C. & 433,476 & Eskildsen, M.R. & 433,476 \\
\hline Andersen, N.H. & 433,476 & Capan, C. & 307 & Esposito, E. & 645 \\
\hline Ando, $K$. & 1051 & Chanda, B. & 295 & Estovez-Rams, E. & 549 \\
\hline Antipov, E.V. & 113,137 & Choong, S.W. & 275,936 & Etienne, B. & 307 \\
\hline
\end{tabular}


Ezerskaya, E.V. $\quad 893$

Farbod, M. $\quad 129$

Farzaneh, M. 175

Fathy, D. $\quad 601$

Femandes, A.A.R. 861

Fortman, E.L. $\quad 1023$

Fortman, E.L. $\quad 1023$

Finkel, V.A. $\quad 312$

Fisher, I.R. $\quad 433$

Fisher, L.M. $\quad 515$

Frunzio, L. $\quad 645$

Fruchart, D. $\quad 737,991$

Gadjiev, G.M. $\quad 961$

Gaiora, R.M. $\quad 727$

Gammel, P.L. $\quad 433,476$

Garg, K.B. $\quad 327$

Geibel, C. $\quad 371$

Ghazi. M.E. $\quad 275,936$

Ghomineztad, M. $\quad 1099,1107$

Ghorbanzadeh, A. 837

Giorgetti, Ch. $\quad 727$

Gnatchenko, S.L. 237

Goomert, P. 1122

Gogadze, G.A. $\quad 355$

Goodilin, E.A. $\quad 167$

Grillo, M.L.N. $\quad 860$

Gurevich, A.M. $\quad 956,967$

Haage, T. $\quad 573$

Habermeier, H.U. 13,16,41.

573,905

Hadjev, V. $\quad 573$

Haga, Y. $\quad 371$

Hamam, Y.A. $\quad 1115$

UH Haq, A. $\quad 1068$

utHaq, E. $\quad 951$

Hardiman, M. $\quad 219$
Hasanain, S.K. $\quad 607,613,951$

Hatton, P.D. $\quad 275,565,936$

Hayrynen. T. 83,145

Hedo, M. $\quad 371$

Heine, G. $\quad 319$

Hino, M. $\quad 817$

Hissa, J. 83, 145

Hock, NG.T. $\quad 598$

Holland, O.W. $\quad 601$

Hosseinpour, A. 1065,1075

Houshiar, M. $\quad 943$

Hudson, T.C. $\quad 565$

Inada, $Y$. $\quad 371$

Islam, A.K.MA. $\quad 75,91,283$

Istam, A.T.M.N. $\quad 75$

Istam, R.S. $\quad 91$

Ismail, A.O. 1115

Ishchenko, L.A. $\quad 956,967$

Iraj-zad, A. $\quad 219$

Ivanchenko, E.A. $\quad 867$

Iwamura, $\mathrm{H} . \quad 809$

Izhyk, E.V. $\quad 677$

Jager, D. $\quad 669$

Jahan-Miri, M. 205

Jamali, J.B. $\quad 809$

Jensen, J. $\quad 707,711$

Jinunturya, N. $\quad 289$

Johansson, B. $\quad 259$

Joob, Ch. $\quad 573$

Kallias, G. $\quad 447$

Kallio, A.

83,145

Kakurai, $\mathrm{K}$.

817

Kameli, $P$.

585

Kamilov, I.K.

$581,899,961$

Kardar, $M$.

529

Kaskamalas, S. $\quad 289$
Kazakov, S.M. $\quad 137$

Khan, A.Q. $\quad 1068$

Khan, M.N. $\quad 153$

Khatsko, E.N. $\quad 801$

Khizar, M. $\quad 153$

Khizriev, K. Sh. $\quad 899$

Khosroabadi, H. 251

Khotkevich, A.V. $\quad 349,768$

Khotkevich, V.V. $\quad 349,768$

Khristenko, E.V. $\quad 401$

Kianvash, A. 1007, 1015, 1037

Kilic, A. $\quad 491$

Kilic, K.C. $\quad 491$

Kinal, A. $\quad 893$

Kirichenko, A.Y. $\quad 677$

Kitazawa, K. $\quad 461$

Knotko, A.V. $\quad 167$

Koh, J.G. $\quad 931$

Kohandel, M. $\quad 385,529$

Korzhavyi, P.A. 259

Kouba, R. $\quad 259$

Kozlovskii, M.P. $\quad 873$

Krunavakarn, B. 289

Kuchin, A.G. $\quad 956$

Kumari, K. $\quad 327$

Kurakov, M.P. $\quad 801$

Kuz'michev, N.D. 267

Kuznetsov, V.I. $\quad 409$

Lang, W. $\quad 319$

Lataifeh, M.S. $\quad 789$

Lebedev, 0.I. 137

Lehlooh, A.F.D. $\quad 789,825$

Lennikov, V.V. $\quad 167$

Lin, W.J. $\quad 565$

Lisitskit, M.P. $\quad 645$ 


\begin{tabular}{|c|c|c|c|c|c|}
\hline Liu, R. & 601 & Nappi, C. & 645 & Ramzi, A. & 536,593 \\
\hline Lukina, M.M. & 855 & Naqib, S.H. & 91 & Rassi, D. & 633,639 \\
\hline Lykov, A.N. & 485 & Naqvi, S.M.M.R. & 831 & Ratner, A.M. & 237 \\
\hline Lyubimov, 0.1. & 681 & Nasseri- & & Raza, S.M. & 831 \\
\hline Lyubimova, 1.0. & 681 & Pouryazdi, M. & 1033 & Revyakina, M.G. & 447 \\
\hline Madaah. & & Naziripour, A. & 477,999 & Rezaii, B. & 189 \\
\hline Hosseini, $H$. & 1007 & Nekvasil, V. & 1059 & Rizvi, Sh. & 831 \\
\hline Mahmood, S.H. & 789,825 & Niarchos, D. & 447 & Rizvi, S.D.H. & 831 \\
\hline Mail, N. & 951 & Noda, C. & 541,549 & Rogalev, A. & 727 \\
\hline Mailly, D. & 307 & Noorbakhsh, M. & 1075 & Rueff, J.P. & 727 \\
\hline Mamodov, V.V. & $195,581,961$ & Obolenskii, M.A. & 447 & Sadeghi, $H$. & 1065 \\
\hline Manzoor, S. & 831 & Oleynikov, N.N. & 167,199 & Safa, M. & 181 \\
\hline Marchatti, P.A. & 51 & Omar, $R$. & 598 & Said, M.R. & 783,1115 \\
\hline Mat Salleh, M. & 598 & Omelyanchouk, & & Saidov, K.S. & 794,1059 \\
\hline Matsuda, K. & 809 & A.N. & 417 & Saini, N.L. & 327 \\
\hline MoCormik, P.G. & 1091 & Onukj, Y. & 371 & Sakai, R. & 1051 \\
\hline Matlenny, M.E. & 931 & Oralndo, M.T.D. & 541,549 & Salamati, $H$. & 181,585 \\
\hline Mohta, A. & 775 & Orel, E.S. & 881 & Santiso, J. & 565 \\
\hline Mohammadi & & Pagano, S. & 645 & Sanyal, B. & 775 \\
\hline zadeh, M.R. & 251 & Pamuk, H.O. & 893 & Savel'ev, S.E. & 515 \\
\hline Mookerjoe, A. & 753,775 & Parlato, L. & 645 & Sebt, A. & 1099,1107 \\
\hline Mortensen, $\mathrm{K}$. & 433,476 & Peluso, G. & 645 & Sodighiani, A. & 175 \\
\hline Moshfogh, A.Z. & 585 & Pepe, G.P. & 645 & Selim zadøh, R.l. & 303 \\
\hline Moskalenko, A.V. & 371 & Perez. & & Senoussi, S. & 536,593 \\
\hline Mucha, J. & 341 & Rodriguez, $F$. & 681 & Seyyed- & \\
\hline Mukai, K. & 809 & Petrusenko, Y.T. & 499 & Reihani, M. & 1007 \\
\hline Mukashev, B.N. & 153 & Piechota, S. & $697,849,855$ & Shabat, M.M. & 669 \\
\hline Mukimov, K.M. & 424 & Pissas, M. & 447 & Shafiekhani, A. & 919 \\
\hline Muroi, $M$. & 1091 & Pourarian, F. & $477,931,975$ & Shahnawaz & 923 \\
\hline Murtazaev, A.K. & 899 & & 999 & Shahzada, S. & 607,613 \\
\hline Nader, A. & 557 & Prentslau, N.N. & 956,967 & Shahzamanian, & \\
\hline Naghshara, $\mathrm{H}$. & 335 & Prodan, A.A. & 447,499 & M.A. & 213,842 \\
\hline Naidyuk, Y.G. & 371 & Pshirkov, J.S. & 137 & Shakeripour, $H$. & 161,523 \\
\hline Najafi, A. & 363 & Putilin, S.N. & 137 & Shamsuzzaman, & \\
\hline Nakamura, Y. & 931 & Ragimov, S.S. & 303 & A.M. & 283 \\
\hline Namiranian, A. & 363 & Rainford, B.D. & 943 & Shapovalov, V.A. & $697,849,855$ \\
\hline
\end{tabular}




\begin{tabular}{|c|c|c|c|c|c|}
\hline Shapovabov, V.V. & $697,849,855$ & Szymczak, $H$. & 849,855 & Wolfers, $P$. & 991 \\
\hline Shiga, M. & 817 & Tagiveva, R.T. & 889 & Wosik, J. & 601 \\
\hline Shinagawa, $\mathrm{K}$. & 1051 & Takouchi, A. & 1051 & Yahaya, M. & 598 \\
\hline Shirokow, M.I. & 689 & Troufik, A. & 536,593 & Yamamoto, E. & 371 \\
\hline Shirozu, G. & 817 & Tirbiyine, A. & 536,593 & Yampol'skii, V.A. & 515,681 \\
\hline Shkbovskj, V.A. & 507 & Tomar, V.S. & 923 & Yanson, I.K. & 371 \\
\hline Shlyakhtin, OA. & 199 & Tretyakov, Y.D. & 167,199 & Yavary, $\mathrm{H}$. & 213 \\
\hline Shlyk, L.V. & 967 & Tulin, V.A. & 409 & Yoksan, S. & 289 \\
\hline Shokri, AA. & 919 & Usatenko, Z.E. & 873 & Yoodbashi & \\
\hline Shuksinov, Y.M. & 363,385 & Vaez-zadeh, S. & 1081 & zadeh, $\mathrm{H}$. & 1007 \\
\hline Singhal, R.K. & 327 & Valiev, U.V. & 794,1059 & Yu, L. & 51 \\
\hline Sohrabpour, S. & 7 & Van- & & Zadeh, H.S. & 923 \\
\hline Soloshenko, A.P. & 167 & Tendeloo, G. & 137 & Zakery, A. & 1065 \\
\hline Solovjow, A.S. & 573 & Vasykov, V. & 855 & Zamanian, M. & 1081 \\
\hline Sokolov, B.Y. & 424 & Velichko, A.V. & 677 & Zareyan, M. & 417 \\
\hline Soroka, A.A. & 507 & Venkatesh, S. & 923 & Zargar- & \\
\hline Soroka, A.K. & 507 & Vesaghi, M.A. & 919 & Shoushtari, M. & 189 \\
\hline Streot, R. & 1091 & Vovk, R.V. & 447 & Zhikol, O.A. & 893 \\
\hline Su, Y. & 936 & Wallaco, W.E. & 999 & Zhuravlev, Y. & 633,639 \\
\hline Su, Z.B. & 51 & Wang, X.L. & 319 & Zolocherskii, I.V. & 401 \\
\hline Suzuld, K. & 809 & Warthmann, R. & 573 & Zolfagharkhani, G. & 175 \\
\hline
\end{tabular}

